\title{
Erratum to: Toward (car)borane-based molecular magnets
}

\author{
Josep M. Oliva ${ }^{1}$ Diego R. Alcoba ${ }^{2,3} \cdot$ Ofelia B. Oña $^{4} \cdot$ Alicia Torre $^{5} \cdot$ Luis Lain $^{5}$. \\ Josef Michl $^{6}$
}

Published online: 10 March 2016

(C) Springer-Verlag Berlin Heidelberg 2016

\section{Erratum to: Theor Chem Acc (2015) 134:9 DOI 10.1007/s00214-014-1611-5}

In the original publication of the article, misleading fragment labels of (car)borane-based molecular compounds appear in the abstract as well as in the text.

In order to build the polyradical carborane systems, we always start with the well-known neutral radical $\mathrm{CB}_{11} \mathrm{H}_{12}$. These units are linked through $-\mathrm{CH}_{2}-$ bridge units, after removing hydrogen atoms from the carborane cages.

The online version of the original article can be found under doi:10.1007/s00214-014-1611-5.

Josep M. Oliva

j.m.oliva@iqfr.csic.es

1 Instituto de Química-Física "Rocasolano", Consejo Superior de Investigaciones Científicas, 28006 Madrid, Spain

2 Departamento de Física, Facultad de Ciencias Exactas y Naturales, Universidad de Buenos Aires, Buenos Aires, Argentina

3 Instituto de Física de Buenos Aires, Consejo Nacional de Investigaciones Científicas y Técnicas, Ciudad Universitaria, 1428 Buenos Aires, Argentina

4 Instituto de Investigaciones Fisicoquímicas Teóricas y Aplicadas, Universidad Nacional de La Plata, CCT La Plata, Consejo Nacional de Investigaciones Científicas y Técnicas, Diag. 113 y 64 (S/N), Sucursal 4, CC 16, 1900 La Plata, Argentina

5 Departamento de Química Física, Facultad de Ciencia y Tecnología, Universidad del País Vasco, Apdo. 644, 48080 Bilbao, Spain

6 Institute of Organic Chemistry and Biochemistry, Academy of Sciences of the Czech Republic, 16610 Prague 6,

Czech Republic
The correct description for fragments and formulae of the carborane polyradicals is given in Table 1 .

Table 1 Fragments, number of fragments (\# Frag), structure (Struc) and formula for the polyradical carborane compounds

\begin{tabular}{|c|c|c|c|}
\hline Fragments & \# Frag & Struc & Formula \\
\hline $\mathrm{CB}_{11} \mathrm{H}_{11}^{*}$ & 2 & Linear & ${ }^{\cdot}\left(\mathrm{H}_{11} \mathrm{~B}_{11} \mathrm{C}\right)-\mathrm{CH}_{2}-\left(\mathrm{CB}_{11} \mathrm{H}_{11}\right)^{\bullet}$ \\
\hline $\begin{array}{l}\mathrm{CB}_{11} \mathrm{H}_{11}^{*} \\
\mathrm{CB}_{11} \mathrm{H}_{10}^{-}\end{array}$ & 3 & Linear & $\begin{array}{l}{ }^{\circ}\left(\mathrm{H}_{11} \mathrm{~B}_{11} \mathrm{C}\right)-\mathrm{CH}_{2}-\left(\mathrm{CB}_{11} \mathrm{H}_{10}\right)^{\circ}- \\
\mathrm{CH}_{2}-\left(\mathrm{CB}_{11} \mathrm{H}_{11}\right)^{\circ}\end{array}$ \\
\hline $\mathrm{CB}_{11} \mathrm{H}_{10}^{\bullet}$ & 3 & Cyclic & {$\left[\left(\mathrm{CB}_{11} \mathrm{H}_{10}\right)^{\bullet}-\mathrm{CH}_{2}-\right]_{3}$} \\
\hline $\mathrm{CB}_{11} \mathrm{H}_{9}^{*}$ & 4 & Tetrahedral & {$\left[\left(\mathrm{CB}_{11} \mathrm{H}_{9}\right)^{*}-\mathrm{CH}_{2}-\right]_{4}$} \\
\hline
\end{tabular}

